\title{
Synchrotron Studies of Quantum Emergence in Non-Low Dimensional Materials Final Report
}

For Period June 1, 2007 through May 31, 2011

\section{Principal Investigator---J.W. Allen}

The University of Michigan

1060 Wolverine Tower

3003 S. State Street

Ann Arbor, MI 48109-1224

Prepared for

The U.S. Department of Energy

Award Number DE-FG02-07ER46379

\section{Table of Contents}

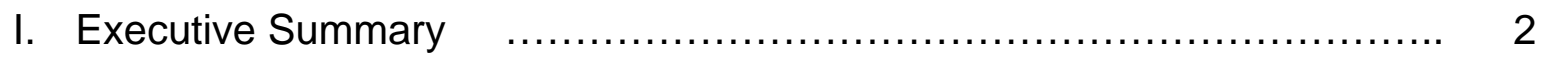

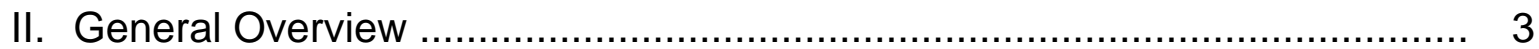

III. Research Results

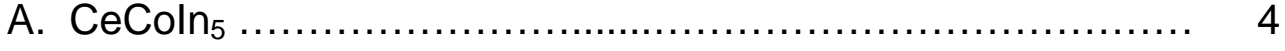

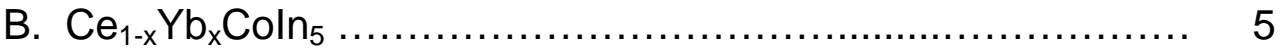

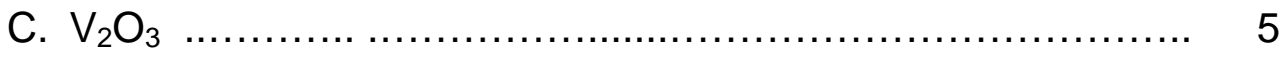

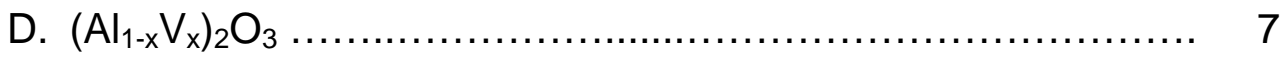

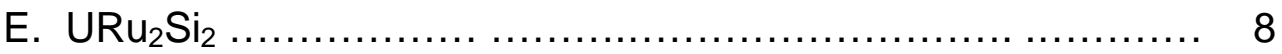

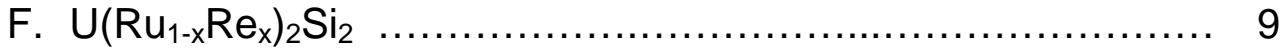

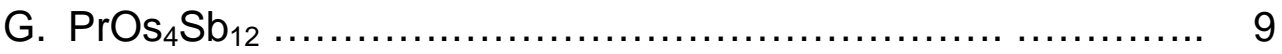

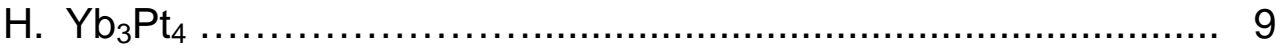

I. Comparison of results achieved with goals and objectives $\quad \ldots . . \quad 9$

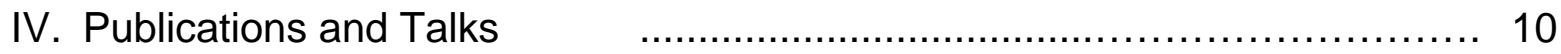

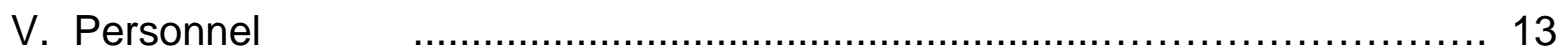

This report was prepared as an account of work sponsored by the United States Government. Neither the United States nor the United States Department of Energy, nor any of their employees, nor any of their contractors, subcontractors, or their employees, makes any warranty, express or implied, or assumes any legal liability or responsibility for the accuracy, completeness, or usefulness of any information, apparatus, product or process disclosed or represents that its use would not infringe privately-owned rights. 


\section{Executive Summary}

Emergence - the tendency of aggregates of interacting individuals to display behaviors that are present only for the aggregate and not for the individuals - is a broad organizing principle of nature that extends across complexity scales ranging from collections of electrons in solids to collections of humans in societies. In spite of the enormous differences in the situations of these two extremes, e.g. that electrons are directly governed by the principles of quantum mechanics and humans manifestly are not, nonetheless the former has great relevance for the latter because it offers the possibility of understanding nontrivial emergent phenomena at a level of quantitative or semi-quantitative detail that can be elusive in the more complex systems. Studies of quantum emergence in condensed matter physics contribute to the larger effort of human scientific inquiry as a provider of paradigms of emergent behaviors and at the same time increase understanding at the forefront of the science of materials.

This project aims to investigate the quantum mechanical behaviors of electrons in materials for which emergence is particularly important because their properties cannot be described simply in terms of the properties of a collection of non-interacting electrons. The term "non-low dimensional" excludes materials where such properties are directly the result of arrangements of atoms in the solid that are essentially linear or planar. Non-low dimensional materials that display emergence tend to have transition metal, rare earth or actinide elements as constituents because electrons from the atomic $d$ and $f$ shells of these elements tend to interact very strongly in the solid. These interactions, and competition between different ones of them, give rise to the emergent behaviors, particularly when the competition causes the material to be poised between two collective states like magnetism and superconductivity, a situation called a "quantum critical point." Magnetism and superconductivity are useful in technology and in general, an understanding of actinide materials is considered important in connection with the nation's defense and security. Thus understanding these materials is important in both basic and applied science, although this project is focused on the former.

The largest part of the experimental work has used photons, obtained primarily at synchrotron facilities, but also in a home laboratory, to excite electrons from a solid sample, a technique known as photoelectron spectroscopy (PES). Detecting the angular and energy dependences of the excited electrons enables the momentum and energy dependences of their quantum mechanical states to be determined. The work takes advantage of recent developments in synchrotron-excited PES for these materials that allow the making of tomographic maps of the momentum dependence for specific energies, somewhat analogous to making contour maps in geography. The immediate goal of the work has been to obtain maps with exceptionally high resolution and clarity for the particular materials chosen for study. Such quality in the experimental maps is required to adequately test theoretical maps calculated by collaborators who use a new method with the technical name "dynamic mean field theory," designed particularly for materials where the electrons interact strongly. 
Research of the kind undertaken in this project is producing results that will find their way into the next generation of solid state text books and applied science will be able to draw on the new understanding to better achieve its goals. Graduate students and postdocs performing the proposed research receive excellent training that prepares them for future careers in the science and technology of our nation.

\section{General Overview}

Plan and timing of the report This is the final report of the 4-year grant. The research descriptions are organized by topic.

Objective and technical approach of the project The objective of this work is to determine the single-particle electronic structure of selected non-low dimensional materials and to analyze the data with a view to elucidating the relation between their electronic structures and emergent properties, i.e. properties that are characteristic of the assembled material and not merely of the constituent atoms. Such properties include mixed valence, heavy Fermions, the Kondo effect, insulator-metal transitions, non-Fermi liquid behaviors, quantum criticality, magnetism and superconductivity.

We measure primarily the single particle electronic structures of materials using photoemission spectroscopy (PES). Occasionally we also do x-ray absorption spectroscopy (XAS), resonant X-ray scattering (RXS) and non-resonant inelastic x-ray scattering (NIXS). These data are obtained at synchrotrons and in an in-house laboratory instrument equipped for X-ray photoemission spectroscopy (XPS) and ultraviolet photoemission spectroscopy (UPS). The synchrotron work is of three main types, (1) resonant angle-integrated PES at a variety of photon energies to identify and extract the spectrum of the narrow band electrons of the material, (2) high resolution, low temperature, angle-resolved photoemission spectroscopy (ARPES) to determine the crystal momentum (k) dependence of the hole excitations of a single crystal, and (3) the making k-dependent intensity maps for fixed binding energies, especially for zero binding energy which produces a Fermi surface (FS) map. All these data are analyzed in the home laboratory by comparison to theoretical results from local density-functional (LDA) calculations, from solutions of model many-body Hamiltonians, from phenomenological single electron self-energies, and most recently to calculated manybody spectra from dynamic mean field theory (DMFT) combined with LDA, i.e. LDA + DMFT.

Synchrotrons and collaborations The experiments were performed by us or collaborators on various beamlines located at the Advanced Light Source (ALS), the Advanced Photon Source (APS), the SPring8 Synchrotron in Japan and at the National Synchrotron Radiation Research Center (NSRRC) in Taiwan. The work benefited greatly from collaborations with Dr. Jonathan Denlinger (ALS), Dr. Robert Gordon (APS), Dr. Christie Nelson (Brookhaven National Lab, working at APS), Prof. L. H. Tjeng (University of Cologne, working at NSRRC), and Professors S. Suga and A. 
Sekiyama (University of Osaka, working at SPring8). Tjeng and Denlinger were once postdocs in our group.

Our main LDA collaborator has been Peter Oppeneer of Upsala University. We have also performed our own LDA calculations using the Wien2K code that we acquired. Our LDA+DMFT collaborators have been K. Haule and G. Kotliar at Rutgers.

With the exception of $\mathrm{Yb}_{3} \mathrm{Pt}_{4}$ obtained from Prof. Meigan Aronson at the Brookhaven National Laboratory, samples of materials containing $\mathrm{Ce}, \mathrm{Yb}$, and $\mathrm{U}$ were obtained from Prof. Brian Maple at the University of California at San Diego (UCSD). Samples of $\mathrm{V}_{2} \mathrm{O}_{3}$ were obtained from Dr. Patricia Metcalf at Purdue University. Samples of $\left(\mathrm{Al}_{1-\mathrm{x}} \mathrm{V}_{\mathrm{x}}\right)_{2} \mathrm{O}_{3}$ were obtained many years ago from Dr. Tom Read (now deceased) of the M.I.T. Lincoln Laboratory.

\section{Research Results}

\section{A. $\mathrm{CeColn}_{5}$}

The CeTIn 5 ( $T=\mathrm{Co}, \mathrm{Rh}, \mathrm{Ir}$ ) compounds and their binary alloys based on the various $\mathrm{T}$ elements display a complex phase diagram with SC and magnetism and related quantum critical points. Apart from the general notions that the superconductivity and magnetism are somehow related, and that the layered crystal structure plays a role, the specific origin of the SC remains to be elucidated.

In collaboration with Jonathan Denlinger at the ALS, photon energy dependent ARPES data on the heavy fermion superconductor $\mathrm{CeColn}_{5}$, taken at $26 \mathrm{~K}$ in a time period preceding the inception of the grant, were analyzed in detail to obtain the three dimensional Fermi surface. The data have been compared to theoretical three dimensional Fermi surfaces obtained in LDA calculations by Peter Oppeneer, performed both with the $\mathrm{Ce} 4 \mathrm{f}$ electrons treated as localized to the core and as itinerant (part of the band electrons). By accounting for modest broadening in the $\mathrm{k}_{\mathrm{z}}$ component, and by discriminating against data from cleaved surfaces that manifest a strong surface state, excellent agreement is found between experiment and the localized calculation, and very poor agreement is found for the itinerant calculation. This result is significant because the temperature is roughly half of the coherence onset temperature of $45 \mathrm{~K}$, identified in the recent "two fluid analysis" of Nakatsuji, Pines and Fisk as the temperature where a heavy coherent Kondo liquid begins to form. One might expect that this would manifest as a change in the volume of the Fermi surface but our result says that this does not happen. Recent LDA + DMFT theory might be consistent with our result, i.e. that coherence develops at a much higher temperature than for the Fermi surface size change. Further ARPES experiments were done during the period of the grant at temperatures $20 \mathrm{~K}$ to $200 \mathrm{~K}$, with the result that the Fermi surface is essentially the same as determined in detail at $26 \mathrm{~K}$. 


\section{B. $\mathrm{Ce}_{1-\mathrm{x}} \mathrm{Yb}_{\mathrm{x}} \mathrm{Coln}_{5}$}

$\mathrm{Ce}_{1-x} \mathrm{Yb}_{\mathrm{x}} \mathrm{Coln}_{5}$ is a new alloy series having properties that may give a fresh view on the origin of the SC in the $x=0$ compound. A simplification is that there is no evidence of magnetic ordering for any $x$. Transport measurements find that the $x=0$ SC persists up to $x=0.65$. A two phase region exists from $x=0.8$ to $x=1$ but with no change in crystal structure and only small jumps in lattice constants. Simple metallic resistivity for $x=1$ suggests [1] $\mathrm{Yb}^{2+} 4 \mathrm{f}^{14}$. In contrast the resistivity for all $x<0.8$ shows Kondo lattice behavior with a maximum at a coherence temperature $T_{\text {coh }}$ nearly constant with $x$, motivating the proposal by our UCSD collaborators of a cooperative intermediate valence (CIV) state. In collaboration with Jonathan Denlinger we have characterized the electronic structure by XAS, XPS and ARPES performed at the ALS. From XAS at the $\mathrm{Ce} \mathrm{M}_{4,5}$ edges we determined the Ce valence to be near $3+$ and essentially independent of $x$. The $Y b$ valence from $4 f X P S$ spectra varies from $\approx 2.8$ for $x=0.1$ to $\approx$ 2.3 for $x=1$, supporting the CIV concept and suggesting that $Y^{\prime b C o l n} n_{5}$ may have a very large $T_{\text {coh }}$, analogous to an $\alpha$-like Ce compound. ARPES spectra show that characteristic quasi-two dimensional Fermi surface (FS) electron pockets for $x=0$ shrink considerably for $x=1$, consistent with an atomic electron counting change from $4 \mathrm{f}^{1} 5 \mathrm{~d}^{1} 6 \mathrm{~s}^{2}$ (4 e-/Ce) to $4 f^{13} 5 d^{1} 6 s^{2}(2 e-/ Y b)$ and the Luttinger theorem requirement that the $4 f$ electron $(x=0)$ and the $4 \mathrm{f}$ hole $(x=1)$ be included in the FS because the $4 \mathrm{f}$ magnetic moment is quenched. This FS size variation might correlate with the loss of SC. The jump in properties across the two phase region is reminiscent of the Ce $\gamma-\alpha$ transition except for the very small change in lattice, which is then more suggestive of the not yet understood phase transition in $\mathrm{YbCuln}_{4}$. In any case our results show that the essentially uncorrelated behavior of the $x=1$ compound is due to very fast valence fluctuations rather than a divalent $Y b f^{14}$ configuration.

\section{C. $\mathrm{V}_{2} \mathrm{O}_{3}$}

$\left(\mathrm{V}_{1-\mathrm{x}} \mathrm{T}_{\mathrm{x}}\right)_{2} \mathrm{O}_{3}(\mathrm{~T}=\mathrm{Cr}, \mathrm{Ti})$ is the archetypal Mott metal-insulator transition (MIT) system displaying paramagnetic metallic (PM), paramagnetic insulating (PI) and antiferromagnetic insulating (AFI) phases. The bulk $V 3 d$ PM phase PES spectrum displays at the Fermi energy $E_{F}$ a prominent quasi-particle (QP) peak that is predicted in LDA+DMFT calculations. During the grant period, in collaboration with Jonathan Denlinger and his ALS postdoc Oleg Krupin, we have sought to make ARPES studies of $\mathrm{V}_{2} \mathrm{O}_{3}$ and its alloys to study the QP k-dependence. This is a measurement that had not yet been done successfully by any group using either films or cleaved surfaces.

\section{ARPES on thin films}

One effort was to explore the in situ growth and ARPES measurement of thin films. The main goal was to obtain samples of such high quality as to allow ARPES to be done in the strongly correlated metallic state. Typically no combination of growth conditions, substrate choice, etc., enabled us to obtain films in the metallic state. However one film showed a small bit of metallic weight at the Fermi energy $E_{F}$, and this weight showed 
the metal-insulator transition. Unfortunately this sample was not fully and uniformly metallic and the thin film effort was not continued.

\section{ARPES on cleaved single crystals}

We obtained the first ARPES spectra from the metallic phase of cleaved $\mathrm{V}_{2} \mathrm{O}_{3}$, for both the (0001) and the (10-12) surfaces. Many workers have tried over the years without success for such spectra and this is a true breakthrough. It appears to be the combined result of a several technical improvements in surface preparation and in the ARPES technique, including the use of a very small photon spot to select an optimum place to measure, and a large angle detector that enables a full k-space span of data without any further movement of the sample. The PM phase shows distinct k-resolved $E_{F}$ band crossings and associated Fermi surface features of both hole and electron character. The bulk nature of these data is shown by a strong $k_{z}$ dependence observed by varying the photon energy. PM phase LDA calculations predict a complex FS arising from a multiplicity of $3 d E_{F}$ band crossings in complete disagreement with the ARPES data. LDA+DMFT calculations remove the incorrect LDA multiplicity but also fail badly in giving only a single FS with electron character and the wrong overall FS topology. The dispersions also show signatures of mass renormalization and "kink" and "waterfall" features. These new ARPES data call out for renewed theoretical work on this paradigm MIT system.

Another finding, still not fully confirmed at the end of this project, is that for the antiferromagnetic insulator phase we observe dispersion in the part of the $V 3 d$ spectrum that is nearest the Fermi energy. After some effort we are nearly certain that we can exclude the possibility that the spectra are from some unconverted bit of metallic phase (it is a first order transition) with a metallic spectrum shifted away from the Fermi energy by charging. We have observed in past angle integrated spectra a peaky structure at the top of the valence band and have tentatively assigned the peak as the insulator quasiparticle predicted in DMFT. If the ARPES finding holds up it confirms this assignment and is also very important because the strength of the magnetic insulator quasiparticle is a measure of where the material lies in the continuum between Mott and Slater insulators. An early interpretation might be that $\mathrm{V}_{2} \mathrm{O}_{3}$ is closer to being Slater-like than is now thought.

\section{HAXPES}

In spite of its qualitative success LDA +DMFT for $\left(\mathrm{V}_{1-\mathrm{x}} \mathrm{T}_{\mathrm{x}}\right)_{2} \mathrm{O}_{3}(\mathrm{~T}=\mathrm{Cr}, \mathrm{Ti})$ does not yield an internally consistent description of the MIT. In the comparison between early LDA+DMFT calculations and valence band photoemission spectra, the $U$ value consistent with spectroscopy was found to be too small to allow the MIT for the bandwidth $W$ values found in the LDA calculations for the various phases. This basic tension concerning $U$ has proved to be continuing and pervasive even though the theory has been made more sophisticated. More recent LDA + DMFT studies suggest that an effective trigonal crystal field splitting leads to a redistribution of the orbital populations (known as the orbital selective MIT). Nonetheless $U$ must still be changed through the 
transition. A very interesting alternative concept is that the orbital polarization induced by a slight enhancement of the effective crystal field splitting can reduce the critical value $U_{c}$ (relative to $W$ ) for the transition and thereby facilitate the MIT even though $U$ is unchanged. Thus there are now only two possibilities on the table for $\mathrm{V}_{2} \mathrm{O}_{3}$, either $\mathrm{U}$ or $\mathrm{U}_{\mathrm{c}}$ changes in the MIT.

In collaboration with S. Suga and A. Sekiyama we have made at the Spring-8 synchrotron a state of the art hard X-ray photoemission spectroscopy (HAXPES) study to tackle this problem. Since U/W is known to be much different for the surface and the bulk, bulk sensitive HAXPES with photon energy $\mathrm{h} v=5-8 \mathrm{keV}$ is essential by virtue of its large probing depth $(>50 \AA$ at $h v \approx 5-8 \mathrm{keV})$. The very high quality of our HAXPES spectra relative to that of earlier studies enables a detailed analysis to identify in all three phases the incoherent part of the $\vee 3 d$ spectrum, i.e. the lower Hubbard band that defines $U$ on the photoemission side of $E_{F}$. Thereby we obtain very strong evidence that $U$ stays essentially constant through the MIT, leaving a change in $U_{C} / W$ as the viable scenario for the MIT.

\section{Stability of insulating $\mathrm{V}_{2} \mathrm{O}_{3}$ studied by $X A S$ on $\left(\mathrm{Al}_{1-\mathrm{x}} \mathrm{V}_{\mathrm{x}}\right)_{2} \mathrm{O}_{3}$}

The optical spectra of typical Mott-Hubbard insulators such as $\mathrm{NiO}$ or $\mathrm{Cr}_{2} \mathrm{O}_{3}$ display a series of relatively weak but sharp lines and bands that can be clearly related to the predicted energy levels for the relevant valence state of the magnetic ion, albeit with small differences that can also be clearly related to the effects of weak exchange couplings between the ions in producing spin waves and optical excitons. Thus the optical spectrum of $\mathrm{NiO}$ shows the $\mathrm{Ni}^{2+}$ spectrum and the optical spectrum of $\mathrm{Cr}_{2} \mathrm{O}_{3}$ shows a $\mathrm{Cr}^{3+}$ spectrum. The $\mathrm{Cr}^{3+}$ spectrum can even be tracked continuously from heavily doped ruby, $\mathrm{Al}_{2} \mathrm{O}_{3}: \mathrm{Cr}^{3+}$ to that of $\mathrm{Cr}_{2} \mathrm{O}_{3}$ in the alloy system $\left(\mathrm{Al}_{1-\mathrm{x}} \mathrm{Cr}_{\mathrm{x}}\right)_{2} \mathrm{O}_{3}$. It is then a fascinating fact that the optical spectrum of the insulating state of the prototypical Mott transition system $\mathrm{V}_{2} \mathrm{O}_{3}$ does not show a $\mathrm{V}^{3+}$ optical spectrum, although such a spectrum is observed for $\mathrm{Al}_{2} \mathrm{O}_{3}: \mathrm{V}^{3+}$. It can be speculated that this difference is related to the fact that insulating $\mathrm{V}_{2} \mathrm{O}_{3}$ can be made metallic with modest increases in temperature or pressure whereas this is impossible for $\mathrm{Cr}_{2} \mathrm{O}_{3}$. The PI made a very early attack on this question through optical studies of the alloy system $\left(\mathrm{Al}_{1-\mathrm{x}} \mathrm{V}_{\mathrm{x}}\right)_{2} \mathrm{O}_{3}$ for $\mathrm{x}$ up to about $10 \%$ at which point phase separation takes place. These studies showed $\mathrm{V}^{3+}$ pair lines and also the growth of a very strong infrared band that tends to obscure the $\mathrm{V}^{3+}$ pair lines and that is the precursor to the $\mathrm{V}_{2} \mathrm{O}_{3} \mathrm{AFI}$ absorption edge.

In collaboration with $\mathrm{H}$. J. Tjeng and his student $\mathrm{T}$. Haupricht of the University of Cologne, XAS at the $V L_{2,3}$ edges was performed at the NSRRC (Taiwan) on the same samples as were used for the optical studies. All the spectra clearly show the fingerprint structure of $\mathrm{V}^{3+}$, but it was found that the spectra for $\mathrm{V}_{2} \mathrm{O}_{3}$ are much broader than for $\mathrm{V}^{3+}$ in $\mathrm{Al}_{2} \mathrm{O}_{3}$, in great contrast to the unchanging breadth observed when comparing analogous spectra of $\mathrm{NiO}$ and $\mathrm{Ni}$ in $\mathrm{MgO}$. As presented thus far only in Haupricht's thesis, the interpretation that we give to this result is that both the XAS broadening and the infrared optical edge arise from $V$ site to site charge fluctuations 
whose rather low energy signals the possibility that the Mott Hubbard state of insulating $\mathrm{V}_{2} \mathrm{O}_{3}$ can be made unstable rather easily.

\section{E. $\mathrm{URu}_{2} \mathrm{Si}_{2}$}

$\mathrm{URu}_{2} \mathrm{Si}_{2}$ is well known for its $17.5 \mathrm{~K}$ phase transition to a "hidden order" $(\mathrm{HO})$ state. The $\mathrm{HO}$ has yet to be determined. Theoretical HO scenarios typically require or imply a particular paramagnetic-phase Fermi surface. Thus there is a need both to determine the electronic structure of the material and to test scenarios for the $\mathrm{HO}$ itself. We have performed the following work directed at both of these goals.

\section{ARPES}

The three-dimensional (3D) bulk FS topology of the paramagnetic phase has been measured by photon-dependent ARPES of UHV-cleaved surfaces. Characterization of electronic states arising from the silicon-terminated surface using spatial dependent measurements, surface dosing experiments, and theoretical surface slab calculations, has allowed the identification of the bulk electronic band structure. The bulk FS topology is found to be distinctly different from those of both localized $\mathrm{ThRu}_{2} \mathrm{Si}_{2}$-like f-core LDA calculations $\left(\mathrm{U}^{4+}\right)$ and fully itinerant LDA calculations. Key experimental FS topologies can be matched to itinerant LDA contours with suitable Fermi energy shifts that may signal a $4 \mathrm{f}$ valence different from that in itinerant LDA. The intermediate-sized FS band topologies point to an f-occupation between those of the two LDA calculations. In particular a FS nesting scenario for the $\mathrm{HO}$ developed from the latter is precluded by the experimental FS and a hexadecapole scenario for the HO based on LDA+DMFT that gives essentially localized $\mathrm{U}^{4+}$ will require modification to be compatible with the data. The full k-space 3D Fermi surface, probing multiple Brillouin zones, also provides a larger setting for understanding recent high resolution ARPES and STM studies that probe only a narrow region around the zone center.

\section{Resonant $X$-ray Scattering}

At the APS we performed resonant X-ray scattering (RXS) studies at the $L_{2}$ and $M_{2,3}$ edges through the hidden order transition. Our intent was to test a theory prediction by our Rutgers collaborators of hexadecapole ordering by using a resonance effect that is sensitive to this high order multipole. This experiment was not successful, either because the resonance effect is too weak, or because the ordering involves yet higher multiples. We greatly benefited from collaboration with Christie Nelson (BNL) and Jonathan Denlinger (ALS) in these experiments.

\section{Non-Resonant Inelastic X-ray Scattering}

At the APS we have collaborated with Robert Gordon (ALS) and George Sawatzky (UBC) to perform non-resonant inelastic X-ray scattering (NIXS) which is another 
relatively new technique that can be made sensitive to hexadecapole ordering, in this case by studying the inelastic spectrum around the $\mathrm{O}_{4,5}$ edge at large momentum transfer. We have observed a small change in the spectrum going through the hidden order transition but the data are not adequate to support any definitive conclusion.

\section{F. $\mathrm{U}\left(\mathrm{Ru}_{1-\mathrm{x}} \mathrm{Re}_{\mathrm{x}}\right)_{2} \mathrm{Si}_{2}$}

$\mathrm{URu}_{2-x} \mathrm{Re}_{x} \mathrm{Si}_{2}$ has a quantum critical point (QCP) for $x$ roughly 0.3 , separating the hidden order of $\mathrm{x}=0$ and large moment ferromagnetism. Brian Maple's UCSD group has studied this QCP behavior. Our motivations in working on this system were to access the QCP and to study changes in the FS with $x$. We were able to obtain ARPES spectra. The main effect observed was a shift of $E_{F}$ but up to now we have not been able to devise a scenario that enables a clear correlation with the change in properties with Re alloying.

\section{G. $\mathrm{PrOs}_{4} \mathrm{Sb}_{12}$ ARPES}

In collaboration with Jonathan Denlinger at the ALS photon energy dependent ARPES results for the skutterudite compound $\mathrm{PrOs}_{4} \mathrm{Sb}_{12}$ were obtained. Since $\mathrm{Pr}$ is an $\mathrm{f}^{2}$ compound we expected that interesting insights into f-electron Fermi surfaces would emerge by comparing to what we already know about the $\mathrm{f}^{1}$ case of Ce compounds and the $\mathrm{f}^{3}$ case of $U$ compounds. We analyzed these data by comparison to LDA band calculations done by ourselves and others, and with LDA $+U$ calculations done by others. Unfortunately, even after considerable analysis effort, the quality of the data did not permit meaningful comparison with theory and no further work on this topic was undertaken.

\section{H. $\mathrm{Yb}_{3} \mathrm{Pt}_{4}$}

$\mathrm{Yb}_{3} \mathrm{Pt}_{4}$ is a new unconventional antiferromagnet. Although the heat capacity in the ordered state suggests a gapped system, the electrical resistivity gives unambiguous evidence for a Fermi liquid state through a $\mathrm{T}^{2}$ component having a coefficient whose magnitude is rather large, comparable to values found in heavy electron systems. The magnetic data imply that $\mathrm{Yb}$ is trivalent but the ordered state properties were deemed unusual enough to merit a spectroscopic verification. Synchrotron XPS data do indeed imply $\mathrm{Yb}^{3+}$ so the unusual properties do not arise from valence fluctuations.

\section{Comparison of results achieved with goals and objectives}

The proposal for this project set forth four general scientific issues to be addressed. These are briefly appraised in turn below.

\section{General electronic structure and Fermi surface determination}

The research was very successful in accomplishing this goal for most of the novel materials studied and comparison of the data to theories has moved the understanding 
of these materials forward. The studies on $\mathrm{V}_{2} \mathrm{O}_{3}$ and on $\mathrm{Ce}_{1-\mathrm{x}} \mathrm{Yb}_{\mathrm{x}} I \mathrm{n}_{5}$ were two opportunities seized that were not part of the original proposal. Some proposed materials systems were not studied for lack of time.

\section{Competition between magnetism and the Kondo effect}

The work on $\mathrm{Ce}_{1-\mathrm{x}} \mathrm{Yb}_{\mathrm{x}} \mathrm{Coln}$ led to a critique of one aspect of the two-fluid model, which was one of two main topics proposed for study under this category. But we were not successful with the other main topic, of observing non-trivial effects in following a Fermi surface through a quantum critical point.

\section{Search for quantum criticality and E/T scaling in photoemission lineshapes}

We were not successful in finding a situation amenable to reaching this goal, which remains undone by any group for a non-low dimensional material.

\section{Emergent states of Mott-Hubbard insulators}

The XAS study of $\left(\mathrm{Al}_{1-\mathrm{x}} \mathrm{V}_{\mathrm{x}}\right)_{2} \mathrm{O}_{3}$ was very successful in resolving the puzzle that was posed in the proposal, as described in section $\mathrm{D}$ above.

\section{Publications and Talks}

\section{Papers published}

1. H. Fujiwara, A. Sekiyama, S.-K. Mo, J. W. Allen, J. Yamagushi, G. Funabashi, S. Imada, P. Metcalf, A Higashiya, M. Yabashi, K. Tamasaku, T. Ishikawa and S. Suga, "Evidence for the Constancy of $\mathrm{U}$ in the Mott transition of $\mathrm{V}_{2} \mathrm{O}_{3}$ " Phys. Rev. B 84, 075117 (2011).

\section{Papers in preparation}

1. J. D. Denlinger, Feng Wang, Ravi S. Singh, J. W. Allen, K. Rossnagel, S. Elgazzar, P. M. Oppeneer, V. S. Zapf, and M. B. Maple, "Contracted Fermi surface in the Kondo lattice material CeColn ${ }_{5}$ well below the transport coherence onset temperature," in preparation for Phys. Rev. B.

2. L. Dudy, J. D. Denlinger, L. Shu, M. Janoschek, J. W. Allen and M. B. Maple, "Electronic structure of $\mathrm{Ce}_{1-\mathrm{x}} \mathrm{Yb}_{\mathrm{x}} \mathrm{Coln}_{5}$ from spectroscopy and bulk properties," in preparation for Phys. Rev. B. 
3. J. D. Denlinger. O. Krupin, B. J. Kim, Ravi S. Singh, Feng Wang, J. W. Allen, K. Haule, Kyoo Kim, G. Kotliar, J. L. Sarrao, N. P. Butch and M. B. Maple, "Testing Theoretical Scenarios for the Hidden Order in $\mathrm{URu}_{2} \mathrm{Si}_{2}$ With Three Dimensional Bulk Fermi Surface Mapping," in preparation for Phys. Rev. Letters.

4. Oleg Krupin, J.D. Denlinger, B.J. Kim, J.W. Allen, P. Metcalf, "k-resolved structure of quasiparticle peak and Fermi surface topology of $\mathrm{V}_{2} \mathrm{O}_{3}$, " in preparation for Phys. Rev. Letters.

5. T. Haupricht, H. J. Tjeng and J.W. Allen, "Optical and X-ray Absorption Signatures of Intersite Charge Fluctuations in $\left(\mathrm{Al}_{1-\mathrm{x}} \mathrm{V}_{\mathrm{x}}\right)_{2} \mathrm{O}_{3}$," in preparation for Phys. Rev. Letters.

$\underline{\text { Invited Seminars, Lectures and Conference Presentations }}$

1. J. W. Allen, "ARPES for the 115's: Issues and Prospects," Institute for Complex Adaptive Matter Exploratory Workshop, "115 Materials: A Rosetta Stone for the Kondo Lattice?", Aspen, Colorado, August 4, 2007.

2. J. W. Allen, "CeColn ${ }_{5}$ Small Fermi Surface Observed Below the Transport Coherence Onset Temperature," Dept of Energy, X-ray and Neutron Scattering Contractor's Workshop, Warrenton, VA, Oct. 18, 2007.

3. J. D. Denlinger, "ARPES Mapping of the Fermi Surfaces of Three Dimensional Heavy Fermion Systems," Symposium Talk for the Spring Meeting of the German Physical Society (DPG), Condensed Matter Division, Berlin, February 27, 2008.

4. J. W. Allen, "Angle-resolved Photoemission of CeColn : Detailed Comparison to LDA and LDA+DMFT," Invited Symposium Talk for the March Meeting of the American Physical Society, New Orleans, LA, March 11, 2008.

5. J. W. Allen, "Synergistic Opportunities for ARPES and Theory of Correlated Electron Solids: examples from f and d electron systems," Invited talk for the 20th Annual Workshop on Recent Developments in Electronic Structure Methods, University of Illinois at Urbana-Champaign, June 18-20, 2008.

6. J. W. Allen, "f-electron photoemission, then and now," Invited talk for the Workshop on $5 f$ Electron Systems: Properties, Bands, Correlations, University of Cologne, July 11, 2008.

7. J. W. Allen, "Kondo volume collapse, Kondo resonance, Mott transition in Ce and its Compounds (and $\mathrm{YbInCu}_{4}$ )," Physics Department Guest Lecture, University of Cologne, July 14, 2008.

8. J. W. Allen, "Fermi Surface $4 \mathrm{f}$ Electron Exclusion Well Below the Transport Coherence Onset Temperature in CeColn ${ }_{5}$," Invited symposium talk for the 25th Annual Conference on Low Temperature Physics (LT 25), Amsterdam, August 11, 2008.

9. J. W. Allen, "The correlation problem in the heavy fermions: an overview," Invited talk for Fall 2008 Coordination Meeting of the DOE/BES Computational Materials 
Science Network entitled "Predictive Capabilities for Strongly-Correlated Systems," Oak Ridge National Laboratory, Nov. 14-16, 2008.

10. J. W. Allen, "f-electron ARPES -- Very Interesting But Very Hard," Physics Department Seminar, University of Illinois at Urbana-Champaign, Feb. 20, 2009.

11. J. W. Allen, "Photoemission of Correlated Electron Systems I," Physics Department guest lecture, University of Würzberg, July 14, 2009.

12. J. W. Allen, "Photoemission of Correlated Electron Systems II," Physics Department guest lecture, University of Würzberg, July 16, 2009.

Contributed conference presentations with published abstracts

1. J. D. Denlinger, F. Wang, J. W. Allen, M. B. Maple, S. El-Gassar, P. M. Oppeneer, "Fermi Surface Mapping of $\mathrm{Ce}_{2} \mathrm{Rh}_{8} \mathrm{n}_{8}$," (abstract) http://meetings.aps.org/Meeting/MAR08/Event/77028

2. $\quad$ R. S. Singh, F. Wang, J. W. Allen, J. D. Denlinger, X. N. Lin, Gang Cao, "Fermi Surface Mapping of $\mathrm{Sr}_{4} \mathrm{Ru}_{3} \mathrm{O}_{10}$ using Angle Resolved Photoemission,"(abstract) http://meetings.aps.org/Meeting/MAR08/Event/81646

3. O. Krupin , J. Denlinger , B.J. Kim , Ravi S. Singh , J.W. Allen, "Electronic Band Structure of Metallic Phase $\mathrm{V}_{2} \mathrm{O}_{3}$," (abstract) http://meetings.aps.org/Meeting/MAR09/Event/93495

4. J.W. Allen , B.J. Kim , Ravi S. Singh, O. Krupin , J.D. Denlinger , R.E. Baumbach, M.B. Maple, "Electronic band structure of Pr-based filled skutterudite antimonides," (abstract) http://meetings.aps.org/Meeting/MAR09/Event/98844

5. O. Krupin, J.D. Denlinger, B.J. Kim, J.W. Allen, P. Metcalf, "Electron correlations in the k-dependent electronic structure of metallic $\mathrm{V}_{2} \mathrm{O}_{3}$," (abstract) http://meetings.aps.org/Meeting/MAR10/Event/11870

6. J.D. Denlinger, O. Krupin, B.J. Kim, J.W. Allen, K. Haule, Kyoo Kim, G. Kotliar, J.L. Sarrao, N.P. Butch, M.B. Maple, "Surface State Modification of $\mathrm{XRu}_{2} \mathrm{Si}_{2}$, $X=($ La $, \mathrm{Ce}, \mathrm{Th}, \mathrm{U}), "$ (abstract) http://meetings.aps.org/Meeting/MAR10/Event/119123

7. L. Dudy, J.W. Allen, J. Denlinger, L. Shu, M. Janoschek, R.E. Baumbach, M.B. Maple, Electron-spectroscopy of the heavy fermion alloy $\mathrm{Ce}_{1-\mathrm{x}} \mathrm{Yb}_{\mathrm{x}} \mathrm{Coln}_{5}$," (abstract) http://meeting.aps.org/Meeting/MAR11/Event/137168

8. Jonathan Denlinger, O. Krupin, J.W. Allen, B.J. Kim, K. Haule, Kyoo Kim, G. Kotliar, J.L. Sarrao, N.P. Butch, M.B. Maple, "Quantification of U f-valence in $\mathrm{URu}_{2} \mathrm{Si}_{2}$ from 3D Bulk Fermi Surface Topology," (abstract) http://meeting.aps.org/Meeting/MAR11/Event/139975 


\section{Personnel}

The following personnel have been partially supported by this contract.

J.W. Allen, the PI.

\section{Undergraduates:}

Melany Mioduszewski, May 1, 2010 through Aug. 31, 2010 and Feb. 20, 2011 through May 15, 2011. She obtained a UM B.S. degree in Materials Engineering in May, 2011.

\section{Graduate Students:}

Brandon Smith, UM Physics Graduate Research Assistant from May 1, 2007 through Aug. 31, 2007. Decided to switch to a UM theory group for his Ph.D. thesis work.

\section{Postdocs:}

Ravi S. Singh, Oct. 1, 2007 through Feb. 28, 2009. He is now at the Tata Institute for Fundamental Research in India.

Bum Joon Kim, April 1, 2008 through Jan. 31, 2010. He is now a staff scientist at the Argonne National Laboratory.

Jaeyoung Park, Nov. 1, 2008 through Jan. 15, 2010. He is now employed by Nanometrics, Inc. of Milpitas, California.

Lenart Dudy, March 1, 2009 through May 31, 2011. He is currently considering other possible postdoc positions. 